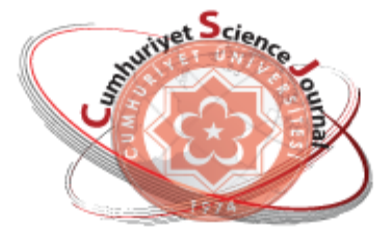

e-ISSN: $2587-246 X$

ISSN: $2587-2680$

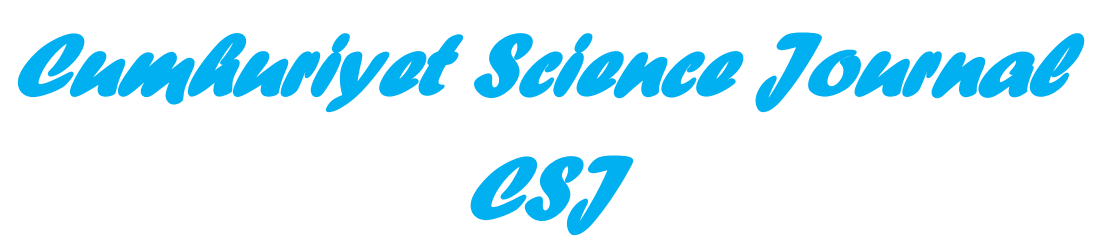

Cumhuriyet Sci. J., Vol.40-4 (2019) 867-874

\title{
An Extension Theorem for Weighted Ricci Curvature on Finsler Manifolds
}

\author{
Yasemin SOYLU ${ }^{1}$ \\ ${ }^{1}$ University of Giresun, Department of Mathematics, Giresun, Turkey
}

\begin{abstract}
Let $(M, F)$ be a forward complete and connected Finsler manifold of dimensional $n \geq 2$. In this study, we extend Wan's extension theorem in Riemannian manifolds to Finsler manifolds by using the weighted Ricci curvature Ric $c_{N}$ bounded below. The proof of theorem is obtained by the Laplacian comparison theorem on Finsler manifolds and the excess function.
\end{abstract}

Keywords: Distance function, Finsler manifold, Weighted Ricci curvature.

\section{Finsler Manifoldlar Üzerinde A ğırlıklı Ricci Eğriliği İçin Bir Genişleme Teoremi}

Özet. (M,F) tam ve bağlantılı $\mathrm{n} \geq 2$ boyutlu bir Finsler manifold olsun. $\mathrm{Bu}$ çalışmada aşağıdan sınırlı $\mathrm{Ric}_{N}$ ağırlıklı Ricci eğriliği yardımıyla Wan'ın Riemann manifoldlarında elde ettiği genişleme teoremi Finsler manifoldlara genişletilmiştir. Teoremin ispatı Finsler manifoldlar üzerindeki Laplasyan karşılaştırma teoremi ve excess fonksiyonu kullanılarak elde edilmiştir.

Anahtar Kelimeler: Uzaklık fonksiyonu, Finsler manifold, Ağırlıklı Ricci eğriliği.

\section{INTRODUCTION}

Finsler geometry includes analogues for many of the natural objects in Riemannian geometry. It is just Riemannian geometry without quadratic restriction. The recent works have shown that some results in Riemannian geometry have been extended to the Finsler setting. For example in this scope, the reader is referred to $[1,2,3]$ and references therein.

In the Riemannian case, in [4], Myers obtained a compactness theorem. The theorem of Myers concludes that if Ric $\geq(n-1) K>0$, then $\operatorname{diam}(M) \leq \pi / \sqrt{K}$. Later, Calabi [5] extended this theorem as follows:

Theorem 1. Let $M$ be a complete and connected n-dimensional Riemannian manifold with non-negative Ricci curvature. Suppose there exists a point $p_{o} \in M$ such that every geodesic ray $\gamma(t)$ issuing from $p_{o}$ satisfies

$$
\limsup _{k \rightarrow \infty}\left(\int_{0}^{k} \operatorname{Ric}(s)^{1 / 2} d s-1 / 2 \ln (k)\right)=\infty
$$

then manifold is compact.

In [6] Cheeger-Gromov-Taylor have proved the following result: 
Theorem 2. Let $M^{n}$ be a complete and connected Riemannian manifold. If there exist $o \in M$ and $r_{0}, v>$ 0 such that

$$
\operatorname{Ric}(x) \geq \frac{(n-1)\left(\frac{1}{4}+v^{2}\right)}{r^{2}}
$$

holds for all $r(x) \geq r_{0}>0$, then $M$ is compact and the diameter is bounded from above by $\operatorname{diam}_{0}(M)<$ $r_{0} e^{\pi / v}$, where $r$ is distance function defined with respect to fixed point $o \in M$.

The idea of their proof relies on studying carefully the index form or the second variation.

Recently, Wan [7] gave a complementary extension of Calabi and Cheeger-Gromov-Taylor's theorems by showing that the manifold has no ray issuing from some point.

Theorem 3. Let $(\mathrm{M}, \mathrm{g})$ be a complete n-dimensional Riemannian manifold and satisfy

$$
\operatorname{Ric} \geq \frac{H(n, \ell, \omega)}{(r+\omega)^{\ell}}
$$

for all $\mathrm{r} \geq 0, \omega>0$ and $\ell \geq 2$, where $\mathrm{r}$ is a distance function $\mathrm{r}(\mathrm{x})=\mathrm{d}(\mathrm{x}, \mathrm{o})$ with respect to a fixed point $o \in \mathrm{M}$ and $\mathrm{H}$ is a constant depending on $\mathrm{n}, \ell$ and $\omega$. Then manifold is compact. Here $\mathrm{H}$ can be chosen to equal to $(\mathrm{n}-1) \cdot \omega^{\ell-2} \cdot \frac{(\ell-1)^{\ell}}{(\ell-2)^{\ell-2}}$ for $\ell>2$ and $(\mathrm{n}-1) \cdot\left(1+\frac{\omega}{\varepsilon}\right), \varepsilon>0$ for $\ell=2$.

Qui [8] used the idea of Wan, by using the excess function and $V$-Laplacian comparison theorem, he derived an extension of Bonnet- Myers type theorem with the Bakry-Emery Ricci curvature Ric $c_{V}:=$ Ric $-\frac{1}{2} L_{V} g$. Also, he showed that when the vector field $V$ is the gradient of some smooth function $f$ on $M$, i.e., $V=\nabla f$, if $\mathrm{Ric}_{V}$ has a positive lower bound and $|f|$ is bounded, then manifold is compact. Moreover, for the $m$-Bakry-Emery Ricci curvature Ric $c_{V}^{m}:=\operatorname{Ric}-\frac{1}{2} L_{V} g-\frac{1}{m-n} V^{*} \otimes V^{*}$, he obtained a compactness theorem by using a similar method as in the proof of Wan.

Also, these type theorems and other calculations in Riemannian geometry has been generalized in various direction by a lot of authors $[9,10,11,12]$ and references therein.

Motivated by the above studies, in this paper we will prove the corresponding Wan's above theorem, for the weighted Ricci curvature ${\mathrm{R} i c_{N}}_{N}$ on Finsler manifolds. In particular, we will use the Laplacian comparison theorem on Finsler manifolds and the excess function. Our main result is as follows:

Theorem 4. Let $(\mathrm{M}, \mathrm{F}, \mathrm{d} \mu)$ be a forward complete and connected Finsler manifold of dimension $\mathrm{n}$ with arbitrary volume form and let $r$ be the distance function $r(x)=d(x, 0)$ with respect to a fixed point $o \in$ M. Suppose that the weighted Ricci curvature

$$
\mathrm{Ri} c_{N}:=\mathrm{Ri} c_{\infty}-\frac{S^{2}}{N-n} \geq A \psi(r)
$$

for all $N \in(n, \infty)$ and $A$ is a constant depending on $\psi$ and $N$. Then

- Manifold is compact,

- Let $\epsilon$ and $\delta$ be positive arbitrary constants. Then A can be chosen as $\left(\frac{\mathrm{N}-1}{\epsilon}\right)\left(\int_{\epsilon}^{\infty} \psi(\mathrm{s}) \mathrm{ds}\right)^{-1}+\delta$. 
Remark 5. If we choose $\psi=\frac{1}{\mathrm{r}^{2}}$ and $\psi=\frac{1}{(\mathrm{r}+1)^{2}}$, then Theorem 4 becomes Theorem 2 and Theorem 3 in [13], respectively.

Remark 6. If $\psi$ satisfies $\int_{\epsilon}^{\infty} \psi(s) d s=\infty$, then A can be chosen as an arbitrary positive real number.

Choose $\psi=\frac{1}{(r+\omega)^{\ell}}$ such that $\ell \in \mathbb{R}$ and $\omega>0$. So we can obtain the following result:

Corollary 7. Let $(M, F, d \mu)$ be a forward complete and connected Finsler manifold of dimension $n$ with arbitrary volume form and let $r$ be the distance function $r(x)=d(x, 0)$ with respect to a fixed point $o \in$ M. Suppose that the weighted Ricci curvature

$$
\operatorname{Ric}_{N} \geq \frac{B(n, \ell, \omega)}{(r+\omega)^{\ell}}
$$

for all $r \geq 0$ and $\omega>0$, where $r$ is a distance function $r(x)=d(x, 0)$ with respect to a fixed point $o \in M$ and $\mathrm{B}$ is a positive constant depending on $\mathrm{N}, \ell$ and $\omega$. Then manifold is compact. Here $\mathrm{B}$ can be chosen to equal to

$$
\begin{aligned}
& \cdot(N-1) \cdot \omega^{\ell-2} \cdot \frac{(\ell-1)^{\ell}}{(\ell-2)^{\ell-2}}+\delta \text { for } \ell>2, \\
& \cdot \frac{(N-1)(\ell-1)}{\epsilon} \cdot(\omega+\epsilon)^{\ell-1}+\delta \text { for } 1<\ell \leq 2,
\end{aligned}
$$

- an arbitrary positive real number for $\ell \leq 1$

( $\epsilon, \delta$ are positive constants).

Additionally, if $\operatorname{Ric}_{N} \geq \delta>0$, we can rescale the metric such that $\delta$ is bigger than the right hand of (5). So manifold is compact (see [13]). By taking $\psi \equiv$ constant in Theorem 4 we have

Corollary 8. Let $(\mathrm{M}, \mathrm{F}, \mathrm{d} \mu)$ be a forward complete and connected Finsler manifold of dimension $\mathrm{n}$ with arbitrary volume form. Assume that $\operatorname{Ric}_{N} \geq(\mathrm{N}-1) \mathrm{C}, \mathrm{C}>0$, then manifold is compact and the diameter has an upper bound.

The proof of Corollary 8 is almost the same steps of Theorem 2 in [13]. So, it may be omitted here.

Now, we review below a summary of the basic concepts associated with the Finsler geometry.

\section{FINSLER GEOMETRY}

Let $M$ be a differentiable n-manifold and TM be the tangent bundle on $M$, where $T_{x} M$ is tangent space at $\mathrm{x} \in \mathrm{M}$. Set $\mathrm{T}_{0} \mathrm{M}=\mathrm{TM} \backslash\{0\}$. Let $\pi: \mathrm{TM} \rightarrow \mathrm{M}$ be the natural projection and $(\mathrm{x}, \mathrm{y})$ be a point of $\mathrm{TM}$ such that $\mathrm{x} \in \mathrm{M}$ and $\mathrm{y} \in \mathrm{T}_{\mathrm{x}} \mathrm{M}$.

Definition 9. A Finsler metric $\mathrm{F}: \mathrm{TM} \rightarrow[0, \infty)$ is a $\mathcal{C}^{\infty}$-Finsler structure of $\mathrm{M}$ with the following conditions:

1. $\mathrm{F}$ is $\mathcal{C}^{\infty}$ on $\mathrm{T}_{0} \mathrm{M}$ (Regularity),

2. $F(x, \lambda y)=\lambda F(x, y)$ for all $\lambda>0$ (Positive homogeneity),

3. The $n \times n$ Hessian matrix 


$$
\mathrm{g}_{\mathrm{ij}}:=\frac{1}{2}\left[\mathrm{~F}^{2}\right]_{\mathrm{y}^{\mathrm{i}} \mathrm{j}}
$$

is positive-definite at every point of $\mathrm{T}_{0} \mathrm{M}$ (Strong convexity).

The pair (M, F) is called a Finsler manifold.

The Chern curvature $R^{V}$ for vectors fields $X, Y, Z \in T_{X} M \backslash\{0\}$ is defined by

$$
\mathrm{R}^{\mathrm{V}}(\mathrm{X}, \mathrm{Y}) \mathrm{Z}:=\nabla_{\mathrm{X}}^{\mathrm{V}} \nabla_{\mathrm{Y}}^{\mathrm{V}} \mathrm{Z}-\nabla_{\mathrm{Y}}^{\mathrm{V}} \nabla_{\mathrm{X}}^{\mathrm{V}} \mathrm{Z}-\nabla_{[\mathrm{X}, \mathrm{Y}]}^{\mathrm{V}} \mathrm{Z}
$$

In the Riemannian case this curvature does not depend on $V$ and coincides with the Riemannian curvature tensor. The flag curvature is defined as follows:

$$
\mathrm{K}(\mathrm{V}, \mathrm{W}):=\frac{\mathrm{g}_{\mathrm{V}}\left(\mathrm{R}^{\mathrm{V}}(\mathrm{V}, \mathrm{W}) \mathrm{W}, \mathrm{V}\right)}{\mathrm{g}_{\mathrm{V}}(\mathrm{V}, \mathrm{V}) \mathrm{g}_{\mathrm{V}}(\mathrm{W}, \mathrm{W})-\mathrm{g}_{\mathrm{V}}(\mathrm{V}, \mathrm{W})^{2}}
$$

where $\mathrm{V}, \mathrm{W} \in \mathrm{T} \mathrm{x} \backslash\{0\}$ are linearly independent vectors. Then the Ricci curvature of $\mathrm{V}$ (as the trace of the flag curvature) is given as

$$
\operatorname{Ric}(\mathrm{V}):=\sum_{\mathrm{i}=1}^{\mathrm{n}-1} \mathrm{~K}\left(\mathrm{~V}, \mathrm{E}_{\mathrm{i}}\right),
$$

where $\left\{E_{1}, E_{2}, \ldots, E_{n-1}, V / F(V)\right\}$ is an orthonormal basis of $T_{x} M$ with respect to $g_{V}$, namely $g_{V}\left(E_{i}, E_{j}\right)=$ $\delta_{i j}$ and $g_{V}\left(V, E_{i}\right)=0$ for all $i, j=1, \ldots, n-1$.

Let $d \mu=\sigma_{F}(x) d x^{1} d x^{2} \ldots d x^{n}$ be the volume form on $M$. For a vector $V \in T_{x} M \backslash\{0\}$,

$$
\tau(x, V):=\ln \frac{\sqrt{\operatorname{det}\left(g_{i j}(x, V)\right)}}{\sigma_{F}(x)}
$$

is a scalar function on $\mathrm{T}_{\mathrm{X}} \mathrm{M} \backslash\{0\}$ which is called the distortion of $(\mathrm{M}, \mathrm{F}, \mathrm{d} \mu)$. We say that the distortion $\tau$ is a $\mathcal{C}^{\infty}$-function, if $M$ is a Riemannian manifold. Setting

$$
\mathrm{S}(\mathrm{x}, \mathrm{V}):=\left.\frac{\mathrm{d}}{\mathrm{dt}}(\tau(\gamma(\mathrm{t}), \dot{\gamma}(\mathrm{t})))\right|_{\mathrm{t}=0}
$$

where $\gamma$ is the geodesic with $\gamma(0)=x, \dot{\gamma}(0)=V . S(x, \lambda V)=\lambda S(x, V)$ for all $\lambda>0$. S is a scalar function on $\mathrm{T}_{\mathrm{x}} \mathrm{M} \backslash\{0\}$ which is called the $\mathrm{S}$-curvature. From the definition, it seems that the S-curvature measures the rate of change in the distortion along geodesics in the direction $V \in T_{x} M$.

For all $N \in(n, \infty)$, we define the weighted Ricci curvature of $(M, F, d \mu)$ as follows (see [1]):

$$
\left\{\begin{array}{l}
\operatorname{Ric}_{N}(W):=\operatorname{Ric}(W)+\dot{S}(W)-\frac{S(W)^{2}}{N-n} \\
\operatorname{Ric}_{\infty}(W):=\operatorname{Ric}(W)+\dot{S}(W), \\
\operatorname{Ric}_{n}(W):= \begin{cases}\operatorname{Ric}+\dot{S}(W), & \text { if } S(W)=0 \\
-\infty & \text { otherwise. }\end{cases}
\end{array}\right.
$$

Also $\operatorname{Ric}_{N}(\beta W):=\beta^{2} \operatorname{Ric}_{N}(W)$ for $\beta>0$. 
We say that $(\mathrm{M}, \mathrm{F})$ is forward complete if each geodesic $\gamma:[0, \ell] \rightarrow \mathrm{M}$ is extended to a geodesic on $[0, \infty)$, in other words, if exponential map is defined on whole TM. Then the Hopf-Rinow theorem gives that every pair of points in $\mathrm{M}$ can be joined by a minimal geodesic.

The Legendre transformation $\mathcal{L}: \mathrm{TM} \rightarrow \mathrm{T}^{*} \mathrm{M}$ is defined as

$$
\mathcal{L}(\mathrm{Z}):= \begin{cases}\mathrm{g}_{\mathrm{Z}}(\mathrm{Z}, .), & \mathrm{Z} \in \mathrm{T}_{0} \mathrm{M} \\ 0 & \mathrm{Z}=0 .\end{cases}
$$

For a smooth function $h: M \rightarrow \mathbb{R}$, the gradient vector of $h$ at $x \in M$ is defined as $\nabla h(x):=\mathcal{L}^{-1}(d h)$.

Given a smooth vector field $\mathrm{Z}=\mathrm{Z}^{\mathrm{i}} \partial / \partial \mathrm{x}^{\mathrm{i}}$ on $\mathrm{M}$, the divergence of $\mathrm{Z}$ with respect to an arbitrary volume form $\mathrm{d} \mu=\mathrm{e}^{\varphi} \mathrm{dx}^{1} \mathrm{dx}^{2} \ldots \mathrm{dx} \mathrm{x}^{\mathrm{n}}$ is defined by

$$
\operatorname{divZ}:=\sum_{\mathrm{i}=1}^{\mathrm{n}}\left(\frac{\partial \mathrm{Z}^{\mathrm{i}}}{\partial \mathrm{x}^{\mathrm{i}}}+\mathrm{Z}^{\mathrm{i}} \frac{\partial \varphi}{\partial \mathrm{x}^{\mathrm{i}}}\right) .
$$

Then we define the Finsler-Laplacian of $\mathrm{h}$ by $\Delta \mathrm{h}:=\operatorname{div}(\nabla \mathrm{h})=\operatorname{div}\left(\mathcal{L}^{-1}(\mathrm{dh})\right)$.

Let $U=\left\{x \in M:\left.\nabla u\right|_{x} \neq 0\right\}$. The Hessian of $u$ on $U$ is defined by follows:

$$
\mathrm{H}(\mathrm{u})(\mathrm{V}, \mathrm{W}):=\mathrm{VW}(\mathrm{u})-\nabla_{\mathrm{V}}^{\nabla \mathrm{u}} \mathrm{W}(\mathrm{u}), \quad \forall \mathrm{V},\left.\mathrm{W} \in \mathrm{TM}\right|_{\mathrm{U}} .
$$

We know that $\mathrm{H}(\mathrm{u})$ is symmetric, and it can be rewritten as

$$
\mathrm{H}(\mathrm{u})(\mathrm{V}, \mathrm{W})=\mathrm{g}_{\nabla \mathrm{u}}\left(\nabla_{\mathrm{V}}^{\nabla \mathrm{u}} \nabla \mathrm{u}, \mathrm{W}\right) .
$$

The following lemma will be very useful in the proofs of our main results (see [14]).

Lemma 10. Let $(M, F, d \mu)$ be a Finsler n-manifold, and $u: M \rightarrow R$ a smooth function on $M$. Then on $U=$ $\left\{\mathrm{x} \in \mathrm{M}:\left.\nabla \mathrm{u}\right|_{\mathrm{x}} \neq 0\right\}$ we have

$$
\Delta \mathrm{u}=\sum_{\mathrm{i}} \mathrm{H}(\mathrm{u})\left(\mathrm{E}_{\mathrm{i}}, \mathrm{E}_{\mathrm{i}}\right)-\mathrm{S}(\nabla \mathrm{u}):=\operatorname{tr}_{\nabla \mathrm{u}} \mathrm{H}(\mathrm{u})-\mathrm{S}(\nabla \mathrm{u}),
$$

where $E_{1}, E_{2}, \ldots, E_{n}$ is a local $g_{\nabla u}$-orthonormal frame on $U$.

Finally, define revesibility $\lambda:=\lambda(\mathrm{M}, \mathrm{F})$ as follows:

$$
\lambda:=\sup _{x \in M, y \in T M \backslash 0} \frac{F(x,-y)}{F(x, y)} .
$$

It is clear that $\lambda \in[1, \infty]$, and $\lambda=1$ if and only if $(\mathrm{M}, \mathrm{F})$ is called reversible.

\section{THE PROOF OF THE MAIN RESULT}

Let $(M, F, d \mu)$ be a Finsler manifold of dimensional $n$ and $r(x)=d(o, x)$ be a distance function with respect to a fixed point $o \in M$. It is well known that $r$ is only smooth on $M-\left(C_{o} \cup\{o\}\right)$ where $C_{o}$ is the cut locus of the point $o \in M$. We assume that $\sigma$ is a minimal unit speed geodesic segment. We have $\nabla r=$ $\sigma^{\prime}(t)$ in the adapted coordinates with respect to the $r$, and the distance function $r$ satisfies $F(\nabla r)=1$ for all $p \in M-\left(C_{o} \cup\{o\}\right)$ (see [15]). On the other hand, using the Finsler metric we obtain a weighted Riemannian metric $g_{\nabla r}$. Thus we apply the Riemannian calculation for $g_{\nabla r}\left(\right.$ on $\left.M-\left(C_{o} \cup\{o\}\right)\right)$.

In order to prove the Theorem 4 we first give an upper estimate for the Laplacian of the distance function $r(x)=d(o, x)$. 
Lemma 11. [16] If $\operatorname{Ric}_{N} \geq 0$ for $N \in(n, \infty)$, then the Laplacian of the distance function $r(x)=d(0, x)$ from any given point $o \in M$ can be estimated as follows:

$$
\Delta r \leq \frac{N-1}{r}
$$

in the sense of distributions on $\mathrm{M} \backslash\{0\}$.

Now, we can prove our main theorem using the above notations.

Proof of Theorem 4. Let $\sigma(\mathrm{t})$ be a unit speed ray starting from a fixed point $o \in \mathrm{M}$ with $\sigma(0)=0$. For every $\mathrm{t}>0, \Delta \mathrm{r}(\gamma(\mathrm{t}))$ denotes the Finsler-Laplacian of distance function $\mathrm{r}$ from a fixed point $\mathrm{o} \in \mathrm{M}$. It satisfies $F(\nabla r)=1$. In the Finsler case, recall that the Bochner-Weitzenböck formula [17] for a smooth function $\mathrm{u} \in \mathcal{C}^{\infty}(\mathrm{M})$

$$
0=\Delta^{\nabla u}\left(\frac{F(\nabla u)^{2}}{2}\right)=\operatorname{Ri} c_{\infty}(\nabla u)+D(\Delta u)(\nabla u)+\left\|\nabla^{2} u\right\|_{H S(\nabla u)}^{2} .
$$

From the Bochner formula applied to distance function $r$ and by Lemma 10, we have, on $M-\left(C_{p} \cup\{p\}\right)$,

$$
\begin{aligned}
& 0=\operatorname{Ric}_{\infty}(\nabla r)+D(\Delta r)(\nabla r)+\left\|\nabla^{2} r\right\|_{H S(\nabla r)}^{2} \\
& \geq \operatorname{Ric}_{\infty}(\nabla r)+g_{\nabla r}\left(\nabla^{\nabla r} \Delta r, \nabla r\right)+\frac{1}{n-1}\left(t r_{\nabla r} \mathrm{H} e s s r\right)^{2} \\
& =\operatorname{Ric}_{\infty}(\nabla r)+g_{\nabla r}\left(\nabla^{\nabla r} \Delta r, \nabla r\right)+\frac{(\Delta r+S(\nabla r))^{2}}{n-1} .
\end{aligned}
$$

Using the basic inequality

$$
(x+z)^{2} \geq \frac{1}{v+1} x^{2}-\frac{1}{v} z^{2}
$$

holding for all real numbers $x, z$ and positive real number $v$, we have

$$
\frac{(\Delta r+S(\nabla r))^{2}}{n-1} \geq \frac{(\Delta r)^{2}}{(n-1)(v+1)}-\frac{(S(\nabla r))^{2}}{(n-1) v} .
$$

In the case where $N>n$, taking $v=\frac{N-n}{n-1}>0$, (17) yields

$$
\begin{aligned}
& 0 \geq \operatorname{Ric}_{\infty}(\nabla r)+g_{\nabla r}\left(\nabla^{\nabla r} \Delta r, \nabla r\right)+\frac{(\Delta r)^{2}}{N-1}-\frac{(S(\nabla r))^{2}}{N-n} \\
& =\operatorname{Ric}_{N}(\nabla r)+\frac{\partial}{\partial r}(\Delta r)+\frac{(\Delta r)^{2}}{N-1} .
\end{aligned}
$$

Integrating the inequality (19) over the interval $[\epsilon, t]$, we get

$$
0 \geq \int_{\epsilon}^{t} \operatorname{Ric}_{N}(\nabla r) d s+\Delta r(t)-\Delta r(\epsilon)+\frac{1}{N-1} \int_{\epsilon}^{t}(\Delta r)^{2} d s
$$

for any $\epsilon>0$. Because Ric $_{N} \geq A \psi(r)>0$, by Lemma 11, we have $\Delta r(\sigma(t)) \leq \frac{N-1}{t}$.

Now, let $r_{1}(x)=d(o, x)$ and $r_{2}(x)=d(\sigma(j), x)$. We can think of the excess function $e$ as

$$
e(x):=d(o, x)+d(\sigma(j), x)-j,
$$

which measures how much the triangle inequality fails to be an equality. By the triangle inequality, we have $e(x) \geq 0$ and $e(\sigma(t))=0$ for $0 \leq t \leq j$. Thus 


$$
\Delta e(\sigma(t))=\Delta d(o, \sigma(t))+\Delta d(\sigma(j), \sigma(t)) \geq 0
$$

From here, we have

$$
\Delta r(\sigma(t))=\Delta d(\sigma(0), \sigma(t)) \geq-\Delta d(\sigma(j), \sigma(t)) \geq-\frac{n-1}{j-t} .
$$

By taking $j \rightarrow \infty$ in the above inequality, we obtain $\Delta r(\sigma(t)) \geq 0$ and therefore we have

$$
0 \leq \Delta r(\sigma(t)) \leq \frac{N-1}{t}
$$

From (20), (24) and the assumption of Theorem 4, we have

$$
0 \leq \frac{1}{N-1} \int_{\epsilon}^{t}(\Delta r)^{2} d s \leq \frac{N-1}{\epsilon}-\int_{\epsilon}^{t} A \psi(s) d s
$$

We observe that this is a contradiction if $A$ is very large (see [7]). So manifold must be compact. Now we calculate the $A$ constant we need. By taking $t \rightarrow \infty$ and solving. The term

$$
\frac{N-1}{\epsilon}-A \int_{\epsilon}^{\infty} \psi(s) d s \leq 0
$$

we get

$$
A \geq\left(\frac{N-1}{\epsilon}\right)\left(\int_{\epsilon}^{\infty} \psi(s) d s\right)^{-1}
$$

This allows to choose $A=\left(\frac{N-1}{\epsilon}\right)\left(\int_{\epsilon}^{\infty} \psi(s) d s\right)^{-1}+\delta$ for any $\delta>0$. Therefore theorem holds.

Proof of the Corollary 7. If we take $A=B(n, \ell, \omega)$ and $\psi(s)=\frac{1}{(r+\omega)^{\ell}}$ in the proof of the Theorem 4, then we have

$$
\begin{aligned}
& 0 \leq \frac{1}{N-1} \int_{\epsilon}^{t}(\Delta r)^{2} d s \leq \frac{N-1}{\epsilon}-\int_{\epsilon}^{t} \frac{B(n, \ell, \omega)}{(s+\omega)^{\ell}} d s \\
& =\frac{N-1}{\epsilon}-\frac{B}{\ell-1}\left[\frac{1}{(\epsilon+\omega)^{\ell-1}}-\frac{1}{(t+\omega)^{\ell-1}}\right]
\end{aligned}
$$

from the inequality (25). Let $t \rightarrow \infty$. So we get

$$
B \geq(N-1)(\ell-1) \frac{(\epsilon+\omega)^{\ell-1}}{\epsilon} \text {. }
$$

The term $\frac{(\epsilon+\omega)^{\ell-1}}{\epsilon}$ attains its minimal value when $\epsilon=\frac{\omega}{\ell-2}$ for $\ell>2$. Inserting $\epsilon=\frac{\omega}{\ell-2}$ into the above inequality, we have $(N-1) \cdot \omega^{\ell-2} \cdot \frac{(\ell-1)^{\ell}}{(\ell-2)^{\ell-2}}+\delta, \delta>0$. It is easy to see that (ii) for $1<\ell \leq 2$ and (iii) for $\ell \leq 1$.

\section{REFERENCES}

[1] Ohta S., Finsler interpolation inequalities, Calc. Var. Partial Differ. Equ., 36 (2009) 211-249.

[2] Wu B., A note on the generalized Myers theorem for Finsler manifolds, Bull. Korean Math. Soc., 50 (2013) 833-837. 
[3] Yin S., Two compactness theorems on Finsler manifolds with positive weighted Ricci curvature, Results Math., 72 (2017) 319-327.

[4] Myers S.B., Riemannian manifolds with positive mean curvature, Duke Math. J., 8-2 (1941) 401-404.

[5] Calabi E., On Ricci curvature and geodesics, Duke Math. J., 34 (1967) 667-676.

[6] Cheeger J., Gromov M. and Taylor M., Finite propagation speed, kernel estimates for functions of the Laplace operator, and the geometry of complete Riemannian manifolds, J. Differ. Geom., 17-1 (1982) 15-53.

[7] Wan J., An extension of Bonnet-Myers theorem, Math. Z., 291 (2019) 195-197.

[8] Qui H., Extensions of Bonnet-Myers' type theorems with the Bakry-Emery Ricci curvature, https://arxiv.org/abs/1905.01452, (2019).

[9] Lott J., Some geometric properties of the Bakry-Emery-Ricci Tensor, Comment. Math. Helv., 78 (2003) 865-883.

[10] Wei G. and Wylie W., Comparison geometry for the Bakry-Emery-Ricci tensor, J. Differential Geom., 83 (2009) 337-405.

[11] Deshmukh S. and Al-Solamy F.R., Conformal vector fields on a Riemannian manifold, Balkan J. Geom. Appl., 19 (2014) 86-93.

[12] Eker S., The Bochner Vanishing Theorems on the Conformal Killing Vector Fields, TWMS J. App. Eng. Math., 9-1 Special Issue (2019) 114-120.

[13] Soylu Y., Upper Bounds on the Diameter for Finsler Manifolds with Weighted Ricci Curvature, Miskolc Math. Notes, 19-2 (2018) 1173-1184.

[14] Wu B. and Xin Y., Comparison theorems in Finsler geometry and their applications, Math. Ann., 337 (2007) 177-196.

[15] Shen Z., Lectures on Finsler Geometry, World Scientific, Singapore, 2001.

[16] Ohta S. and Sturm K.T., Heat flow on Finsler manifolds, Commun. Pure Appl. Math., 62 (2009) 1386-1433.

[17] Ohta S. and Sturm K.T., Bochner-Weitzenböck formula and Li-Yau estimates on Finsler manifolds, Adv. Math., 252 (2014) 429-448. 